\title{
Coherent Resonant Properties of Cardiac Cells
}

\author{
A. Chorvatova and D. Chorvat Jr \\ International Laser Centre, Bratislava, \\ Slovakia
}

\section{Introduction}

Despite significant advancements in understanding cardiac cell biology, we still lack a clear insight into precise mechanisms that are responsible for the cell functionality. It is becoming increasingly evident that this information does not reside exclusively in the genome or in individual proteins, as no real biological functionality is expressed at these levels. Instead, to comprehend the true functioning of a biological system, it is essential to understand the integrative physiological behaviour of the complex molecular interactions in their natural environment and precise spatio-temporal topology. As more information is available about the living cells, we are uncovering more and more analogies between biological structures and artificially engineered nano/micro devices. We believe that these resemblances are not just coincidence, but that they reflect deep structural and functional relationships of these entities at the mesoscale level.

In this chapter, a new concept for the description of electrically excitable living cardiac cells is presented. Based on an analogy with a laser-like quantum resonator, in this concept each cardiac cell can be represented by a network of independent nodes, having discrete energy levels and certain transition probabilities. The interaction between these nodes is given by a threshold-limited energy transfer in a state analogical to the population inversion, leading to the "laser-like" behaviour of the whole system.

To explain the new concept, we draw a larger picture of the description of living systems, based on their oscillatory behaviour. We present a phenomenon of resonance and debate its eventual role in the synchronisation of the coherent oscillatory behaviour in living systems. We then detail coherent resonant properties of cardiac cells and discuss pulse-generation in the heart based on these properties from an engineering point of view. In the presented framework, the heart is viewed as a coherent network of synchronously firing cardiac cells behaving as pulsed laser-like amplifiers, coupled to pulse-generating pacemaker masteroscillators.

The presented concept emphasizes the study of integrative cellular states and their communication systems from the "engineering" point of view, rather than the simple quantification of protein cascades involved in cell regulation. In parallel, a concept similar to the one described in this chapter can easily be applied to other cell types, such as rhythmically-firing neurones. In light of the novel view of cardiac cells derived from the concept of biological quantum resonators, it is increasingly important to look at cells by assessing their functionality at mesoscopic level, in addition to knowing their composition and structure. Gathered knowledge can also serve for improving existing optoelectronic detection technologies used for biomedical investigation. 


\section{Resonance and living systems}

\subsection{Physiology: Understanding the logic of life}

Physiology, which in Greek means "study of the logic of life", is, in its pure form, an extraordinary discipline which studies the true behaviour of components of complex living systems - such as the coherent functioning of our own heart, capable of pumping blood every second of your life, often for 70 years or more. But what really the life is? What makes human body different from a rock? These questions hunt people for thousands of years and yet, even with recent advancements in the research and technology that brought an outstanding level of knowledge on living systems, we still lack precise definition of what the life really is. In biology, it is generally accepted that the system has to preserve five main features to be considered alive:

1. First, a living system has to have capacity to maintain differences with its environment and thus keep inequality by remaining in a constant movement (example being the electro-chemical gradient guarded by the membrane complexes in living cells).

2. To maintain these differences in a dynamic way, the system needs to insure efficient energy management by constant exchange of energy and materials with the system's environment, leading to the energy transfer and capture (insured, in living cells, by a process known as metabolism).

3. To efficiently minimize energy requirements of a system as a whole, each living system needs to search for appropriate tools to organize its components: in other words, the system needs to compartmentalize its components into sub-systems and specialise their functions (done by creation of organelles and organs).

4. Once compartmentalized, the system has to insure efficient communication between the created specialized sub-systems of the system as a whole, which is secured by advanced information management, in other words by insuring the flow of information within the system's energy-producing units. This extremely important feature of a living system is achieved by efficient stocking and usage of the gathered information (stored in the genetic code of DNA and translated using signalling pathways in cells).

5. Finally, the functioning of a living system requires the ability of an adaptation to a constantly changing environment and therefore its permanent re-engineering. This is guaranteed by the processes of development, reproduction and evolution, which are, in fact, advanced optimization tools used to lower the energy needs required for the system survival (example being genetic mutations).

In this interpretation, living system is a highly energetically-advantageous dynamic disequilibrium of coherently behaving components organized in efficiently communicating sub-systems, which has capacity to adapt to changing environment and reproduce. In other words, in order to stay alive, each system needs to maintain differences with its environment, using wisely its energy by compartmentalization of its tasks and by efficient communication, perpetuating itself by evolutive reproduction. To understand how is such dynamic disequilibrium created and maintained in cells, it is important to comprehend that to keep a system alive, several tools need to be used - the most important of all being an appropriate energy and information management tools. This includes, on one hand, minimization of energy by the permanent search for diversified energy sources and, on the other hand, the transfer of information about the existing energy state, while ensuring a highly orderly behaviour of the network of its subsystems.

In the modern history, one of the first who tried to uncover the relationship between the life and the laws of Physics was Erwin Schrödinger (apart of being a pioneer in the quantum 
mechanics of light) in "What is life?" book (Schrödinger, 1944). In this work, Schrödinger proposed that life is based on an unconventional application of the 2nd thermodynamic law. This principle states that in a non-living world, the entropy of each isolated system which is not in equilibrium will tend to increase over time, while approaching its maximal value in the equilibrium. That is the reason why a wine glass would never spontaneously re-generate from the sand, but if you break it on the beach, it will disintegrate into pieces, which will be shaped by wind and sea, and will eventually turn to sand. In other words the disorder - the entropy - of what was originally the wine glass will increase. In this way, the world is going constantly towards an increase in chaos.

However, while non-living systems are characterized by an increase in entropy that leads to increase in chaos, this principle does not seem to apply to living systems. Instead, these are rather in contrast with the $2^{\text {nd }}$ law of thermodynamics by their effort to always improve their organization and therefore to create an efficient state based on minimal entropy. But what seems to be a paradox at a first sight can actually be explained in a simple way, as living systems always exist as a quasi-opened ones in a much bigger environment, to which the $2^{\text {nd }}$ thermodynamic principle does apply and hence in which the total entropy increases. So, despite the fact that for the period of its lifetime the relative entropy of a living system is decreasing, in the instant of death the system re-equilibrates its electrochemical differences with its environment, reaching a permanent state of thermodynamic equilibrium of "maximum entropy".

To maintain its differences with the environment in a dynamic way, a living system needs to keep its own entropy low in an environment in which entropy is constantly rising and this is done by efficient energy management. Described "paradox" thus explains why every living system has a constant need for energy, as it continuously needs to "fight" against increasing entropy in its environment, which is driving it to engage into a bigger chaos, resulting in death. Maintaining low entropy and therefore high order is a dynamic life-long battle of each and every living creature, which demands efficient energy and information management, leading to synchronous behaviour of its components in harmony with each other in the precise environment.

A system considered alive is characterized by a coherent synchronization of a complex non-linear behaviour of its subsystems, providing the most advantageous energy efficiency. To achieve this aim, it is undeniable that dynamically behaving living systems do function as oscillators: from cell division, circadian cycle to heartbeat, clocklike rhythms are at the bases of functioning of each and every living organism. This means that if we want to keep a system alive, we need to insure that all of its oscillatory components behave coherently in a dynamic disequilibrium and, at the same time, such synergic character of the components of a non-linear living system has to be based on the synchronization of their own non-linear oscillatory behaviour. To understand how a coherent behaviour of a complex oscillatory system is guaranteed, it is necessary to comprehend what drives cyclic behaviour of its components at a first place. Study of synchronous oscillations (described by S. Strogatz (Strogatz, 2003;Strogatz \& Stewart, 1993)) indicates that a coherent behaviour of the system does not grow gradually, but instead it breaks out cooperatively when the number of connections or couplings (even weak ones) between its components suddenly exceeds the threshold. And in the array of different possibilities how to affect such coupling between oscillating components, there is one particular feature: the phenomenon of resonance. 


\subsection{Phenomenon of resonance}

The phenomenon of resonance is known for centuries. It was originally observed in music by the father of music, Ernst Chladni (1756 - 1827), who has done an extensive research on vibrating plates (Chladni, 1787) and, by showing various modes of vibration in a mechanical surface, improved large number of musical instruments. One of the most prominent scientists interested in the phenomenon of resonance was Nikola Tesla (1856-1946), who described its electrical, as well as mechanical versions (Valone, 2002). He ended up obsessed with it, creating resonant lightning storms, artificial earthquakes, or near collapse of a Manhattan skyscraper, which also lead to several inventions, such as the radio prototype. The phenomenon of resonance of light was described by Erwin Schrödinger (Schrödinger, 1933), based on the quantum mechanical principle of electromagnetic propagation in the form of a wave and of a particle, as an eventual "catastrophe" that can happen under certain conditions in the light beam.

Resonance (schematically represented at Fig. 1) is a physical phenomenon, characteristic of oscillatory phenomena and/or systems, such as harmonic oscillators (Bloch, 1997;Bohm, 1951). It is a tendency of an oscillatory system to oscillate at maximum amplitude at specific frequency. Resonance is an abnormally large vibration at moments (and only at the moments) when the frequency of the stimulus is the same, or nearly the same as the natural vibrational frequency of the system. As a result, the system is driven to pick its natural resonance frequency out from a complex excitation, e.g. what we do when we tune a radio to a specific frequency, and it often does it while searching for the best energy efficiency. Consequently, resonance can force systems to take specific shapes and forms, as demonstrate the powerful example of standing wave Chladni figures (Chladni, 1787).

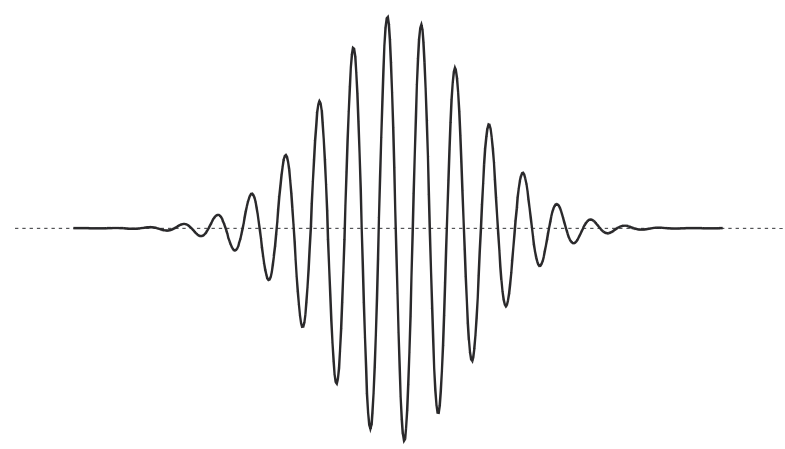

Fig. 1. The pattern of resonance ( $\mathrm{x}$ axis: time, $\mathrm{y}$ axis: amplitude)

Without even realizing it, we often use resonances in our everyday life. Each time you are swinging your child at a swing you do, unconsciously, choose the natural resonance frequency of the system in order to do this task with the smallest effort; each time you tune to your favourite radio, or play an acoustic instrument, you take advantage of resonances. But this phenomenon can also be dangerous, as it can be translated into a non-amortized oscillation reaching the critical frequency of the system and in such a case, the power inside the system rises exponentially. This can lead to oscillating bridges (such as the case of the London Millennium Bridge, phenomenon observed on the day of its opening) (Eckhardt et 
al., 2007), or even their collapse (such as the case of the Tacoma-Narrows bridge) and/or breaking glasses by opera singers.

Resonance can happen in three principal conditions (Bohm, 1951). First, in a specific object, when this object is disturbed at its natural frequency, or the resonance frequency. This situation can happen in mechanical devices, electric circuits, or acoustic instruments. Second, the resonance can build up in an object under conditions when a forcing is done at the same frequency as the natural frequency of the oscillating system. This is an example of the resonance in a pendulum (used when swinging a child on a swing). Finally, third condition arises in the situation of lack of damping or energy loss.

In medicine, resonance technologies are frequently used for the detection of human body alterations during disease, namely the nuclear magnetic resonance (NMR) is well known. Described in 1937, in the theoretical work of I.I. Rabi (Rabi et al., 1992), the method was later applied by Felix Bloch and Edward M. Purcell, who were awarded a Noble Prize of Physics in 1952 for the discovery of new methods for nuclear magnetic precision measurements. This discovery revolutionized medicine by greatly improving non-invasive functional imaging of human body, including the brain and, in 2003, Paul C. Lauterbur and Peter Mansfield were awarded Nobel Prize in Physiology and Medicine for their discoveries in magnetic resonance imaging (MRI).
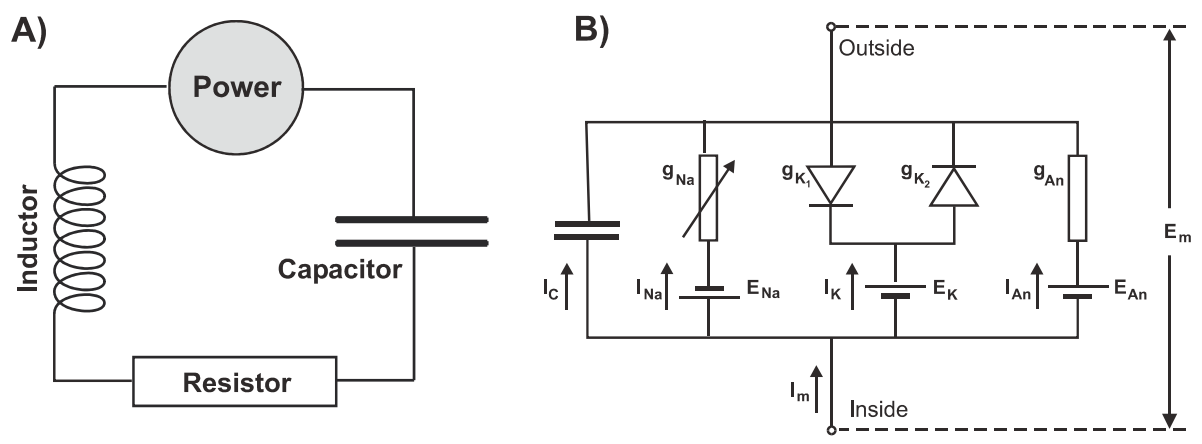

Fig. 2. Comparison of the resonating electric circuit (A) and the equivalent electrical circuit of a cardiac cell (B); I: current, g: conductance, C: capacitance, E: Electric voltage, Na: sodium ions,. K: potassium ions, An: Anions, m: membrane.

Importantly, resonance can happen in any system that uses energy, as each force we know in physics has a resonant representation (Bohm, 1951). Whether it is kinetic, rotational and gravitational energy (the case of pendulum), or electromagnetic one (the case of electrical circuits and lasers), or mechanical and elastic one (the case of resonating bridges), each time when the resonance occurs in a system, the resulting action concerns an energy accumulation. Resonance also occurs in oscillating electrical circuits (Fig. 2A). Each electrical circuit can be described as a resistor-inductor-capacitor (RLC) circuit. If the frequency of power supply of such circuit matches exactly the natural frequency of the circuit's LC combination, the resonance can happen and in such case, the circuit enters the state of resonance. In resonance, the series impedance is minimal and therefore the voltage for a given current is at its minimum. Or, in other words, at resonance, the electric current for a given voltage is at its maximum. It was the original and extraordinary work of Tesla that 
showed us the truly incredible power of resonance in an electrical circuit and can be demonstrated by Tesla electric lightning experiments (Valone, 2002). These experiments, today known as Tesla coils, use conductive bars to direct the lightning and thus can be used to conduct, i.e. to direct the electric signal.

Most of the times, resonance is described in oscillating systems when the oscillator is subjected to periodic forcing as the energetically most efficient state, matching the system's natural frequency of oscillations. Case of a pendulum (Fig. 3) is an example of the resonance using kinetic, elastic, mechanical, gravitational and rotational force.

A

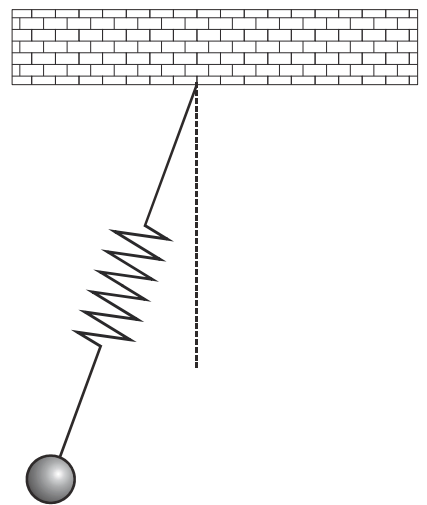

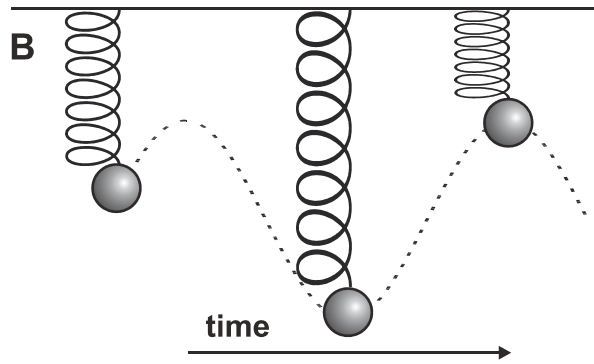

Fig. 3. Schematic representation of a pendulum (A) and its movement in time (B).

Resonance is a state where minimal energy is necessary to induce maximum effect. In other words, when the force that drives an oscillatory system (or its driving force) matches the system's natural frequency of vibrations (also called its resonance frequency), the amplitude of the steady state response will be greatest in proportion to the amount of the driving force. Induced phenomenon of resonance is therefore translated into a tendency of a system to absorb more energy. As a result, resonance state is energetically most efficient state. Consequently, for a system to secure most advantageous energy efficiency, it needs to enter the state of resonance by coherent behaviour of its components.

\subsection{Symphony of life: Resonance in living systems}

The concept of the "symphony of life", based on an idea that life is being played comparably to musical instruments and therefore that there are similarities between the music and functioning of living systems has been known for more than a century. Indeed, already hundred years ago William Bateson used musical analogy to make some evolutionary genetic points clearer and easier to understand as he pointed out that an eight-petal form is the same to a four-petal form as one octave is to another (Bateson, 1894;Bateson, 1913) and there are many other examples. In this understanding, recognition of dynamic patterning process has an important implication for evolutionary thinking. Modularity, segmentation and repetition, observed in living systems, are comparable to measures and tones in music, as pointed out in the Dr Ken Weiss 2002 review on "Good vibrations: the silent symphony of life" (Weiss, 2002). Other researchers identified similarities between Chladni-like simulation and hair-colour patterning (Murray, 1993). Dr Weiss also revealed that chemical "vibration" is harmonious to the organism and has properties similar to those of music (Weiss, 2002). 
Among others, he demonstrated that a small amount of mutational change might have sufficed to reconfigure silent background variation to jump teosine to maize form, creating its most important cultivar - just as Chladni figure can jump when the sound frequency changes.

Another example of comparison between the living system and music was done by Denis Noble in "Music of life" book (Noble, 2006). In this interpretation, functioning of an organ of our body, such as the heart, can be compared to the musical harmony. The book discusses how to reconstruct, at an integrative level, rhythm and more specifically the heart beat, the most obvious of biological oscillators, while analysing how to create a new, higher hierarchical level using regulatory network of interactions at each level of the system organization. Noble's extensive work on the modelling of the beating heart (Noble, 2004) demonstrated that, to understand a complex phenomenon such as the heart rhythm, it is necessary to apply biology that goes beyond the genome. There is no "single gene module" that can explain creation of complex functions such as circadian rhythms, but more gene and protein components appear to be involved.

If life can be described as being played comparably to musical instruments to create a harmonious symphony, it is largely because, as tones of music, all known living systems have oscillatory behaviour. Great majority of processes that are obsered in the living systems function at the bases of an ON - OFF $(0-1)$ "switch-like" states, like musical tonality. This suggests that each system oscillates between these two states at a certain rate, or in other words, with a defined frequency. It can pass from one dynamic state to another at a very short time-base (oscillating frequency-dependent states). Most activities of any known living system (including cells, humans, or populations) work in such a cyclical way. For example, most proteins oscillate between specific states: such as bound/unbound state (for many enzymes), or opened and closed one (for ionic channels, for example), in a synchronous way. And most biochemical regulatory proteins and their ligands interact with other proteins as a lock and a key. Furthermore, we also find oscillatory behaviour at the level of our cells : one of the best examples being cardiac cells, with their capacity to produce periodic oscillations at the frequency of our heart beat. But, in fact, all cells and organisms are subjected to cycles, as nicely described by Arthur T. Winfree in "The Geometry of Biological Time" (Winfree, 1980) or Foster \& Kreitzman in "Rhythms of Life" (Foster R \& Kreitzman L, 2004). We can retrieve such increase-decrease patterns in the functioning of the living system at every of its levels, as demonstrated in Table 1.

\begin{tabular}{|c|c|c|}
\hline Biological effect & & \\
\hline biochemical enzymatic reactions & association & dissociation \\
\hline membrane ionic channels & opening & closing \\
\hline skeletal muscle movement & acceleration & inhibition \\
\hline neuronal networks & stimulation & relaxation (expansion) \\
\hline $\begin{array}{c}\text { cardiac cells } \\
\text { during heart beat }\end{array}$ & contraction & dilatation \\
\hline vessels & constriction & degradation \\
\hline endocrine system & synthesis & reduction $\left(\mathrm{CO}_{2}\right.$ utilization $)$ \\
\hline respiration & oxygenation $\left(\mathrm{O}_{2}\right.$ utilization $)$ & \\
\hline
\end{tabular}

Table 1. Oscillatory phenomena in living systems 
In the last decades, lot of work was done in understanding complex oscillations. Theory that derives from these observations, also referred to as "synchronized chaos", revealed that it is the synergic character of non-linear oscillating systems that make them so rich and powerful. As pointed out by Strogatz (Strogatz, 2003), tendency to synchronize is one of the most general drives in the universe, extending from atoms to animals, from people to planets. Sync is one of the oldest and most elementary parts of non-linear sciences. In this context it is particularly important to understand that it is the synchronization of the chaotic behaviour of oscillatory components which constitutes a complex non-linear dynamic (living) system, and is crucial for the decision-making in choosing the most energetically advantageous interactions of such system with its environment, or between its sub-systems.

As living systems clearly have an oscillatory behaviour and are composed of many subsystems, such oscillatory sub-systems have therefore capacity to generate resonances between themselves. What are scientific proofs that resonance can also occur in living systems? The idea has been around for years: since Georges Lakhovski, who proposed in the "Secret of Life" (Lakhovsky, 1929) that cells can find their resonance frequency of oscillations in an array of multiple vibrations, to a controversial Luc Montagnier, a 2008 Nobel price winner for Medicine for the discovery of HIV virus, who proposed in his disputed article (Montagnier et al., 2009) a surprising idea that resonance can help living systems to recover the memory of events, this issue is now debated for nearly a century. However, this issue remains largely unexplored scientifically and thus still rather debatable with little direct scientific evidence or experimental proof at others than atom and/or molecular levels.

At the molecular level, scientists were clearly able to demonstrate the presence of the resonance energy transfer between atoms and molecules in our cells. In fact, the capacity of Föster resonance energy transfer (FRET) (Lakowicz, 2006;Periasamy, 2001) between atoms and molecules is frequently employed as an imaging method to enhance knowledge on the molecular structure of cellular proteins. Based on visualization of fluorescence which lights up when a resonance transfer occurs between two very close atoms of specific proteins, researchers were capable to establish ultra-structure of great number of proteins, or protein machines (Periasamy et al., 2008). It is also noteworthy that with its ionic channels allowing transmission of ions and creation of the cell membrane potential, each cell in our body is also an electric circuit (Junge, 1992). With an example being the heart cells (see schematic representation of an equivalent electrical circuit for a cardiac cells at Fig. 2B), cell is often an oscillatory electric circuit - and, as described in previous chapters and illustrated at Fig. 2A, resonance can occur in oscillatory electric systems. In addition, a well documented example of the use of resonance in living systems has been found in neuronal networks. This research demonstrated that a living cell has the capacity to generate what is called "stochastic resonance" which is in fact the capability of the cell to extract a specific signal from a large noise (McDonnell \& Abbott, 2009; Wiesenfeld \& Jaramillo, 1998). Stochastic resonance is a cooperative event in which coupling of the oscillatory events of small amplitude and noisy responses improves the system's sensitivity to discriminate weak signals (Moss et al., 2004); thus, the system exhibiting this phenomenon behaves as a kind of detector, trying to extract a weak periodic signal. Presence of resonance in such network makes a difference by allowing a highly efficient extraction of specific signal from a mix of others. 


\subsection{Life as propagator of resonances}

Resonance has all the attributes to act as the driving force in living systems, capable to generate and maintain the highly energetically advantageous dynamic disequilibrium of coherently behaving components crucial for each living system or, more precisely, for the life itself. Examples listed in the Section 2.3 demonstrate the presence of resonance at a molecular level and also suggest that this phenomenon can play a role in the synchronisation of functions in living systems. Taking into consideration the five main features which describe living systems (Section 2.1), resonance can occur in each of them: 1) resonance can happen in oscillating objects with a coherent behaviour that are in constant movement, it therefore allows to maintain differences between the resonating object and its environment in a dynamic way; 2) resonance is a phenomenon directly linked to energy use and maximization, thus allowing efficient energy management; 3 ) confinement of resonance into a distinct space - resonance cavity - pushes each system that employs resonances to compartmentalize and to specialize energy use. 4) The use of resonances is a highly efficient way to retrieve information about the observed system while using minimum energy, as demonstrated by resonance-based sensors and detectors, and thus it also insures an advanced information management. 5) By entering into resonance, oscillating components evolve into a completely new state, based on adaptation with their environment.

Resonance insures highest energy efficiency and helps to understand how the living system can pass from one hierarchical level to another - two fundamental requirements of the living system. As a non-linear effect, resonance has a capacity to energetically boost the synchronized coupling between these components in a precisely determined space. It is a specific state where the system can create maximum energy or, in other words, a state where the system can function with minimum energy. As a result, it can promote cooperativity and thus coherent behaviour of the whole system. In this way, resonance has the ability to push living systems to choose their shape, form, or size (as it is capable to do it in non-living matter when creating standing wave Chladni figures for example) and may thus be a potential decision-maker for these systems. In addition, resonance can only happen between (at least) two coherently oscillating entities - one unit cannot generate resonance. It is a completely new equilibrium, exactly as the creation of a new hierarchical level. As noted by Schrödinger (Schrödinger, 1933), the new state created when two oscillating systems enter the state of resonance is very different form the original states in which these systems were before entering resonance. In this context, resonance is a unique force allowing synchronization of the system, leading to its energetically most efficient coherent behaviour. In this interpretation, life can be understood as based on propagation of resonances, where the life of eukaryotes starts with a resonance between the ovule and the sperm, which then propagates from one cell to another, from one component to another every day of our lifetime, until the resonance transfer weakens and eventually stops. In the latter situation, the system would require much higher amounts of energy to function, engaging in a nonlinear use of energy, leading to a diseased state characterized by an energy collapse and, finally, once the system's natural resonance frequency is perturbed and the system lacks energy to function, the life ends - or, in other words, the living system dies.

\section{Resonant properties of the heart}

\subsection{Coherent resonant properties of cardiac cells}

Previously, our group introduced basic framework for the description of the harmonious behaviour of the heart based on synchronous oscillations of cardiac myocytes, each 
behaving as a pulsed biological "laser-like" quantum resonator/amplifier (Chorvat, Jr. \& Chorvatova, 2008). Already, several other groups emphasized quantum and/or wave features of the behaviour of living biological systems, including studies on conduction pathways in microtubules (Hameroff et al., 2002;Penrose, 2001), DNA mutagenesis (McFadden, 2000;McFadden \& Al Khalili, 1999) and/or information processing (Davies, 2005;Davies, 2004) and its synchronization (Strogatz \& Stewart, 1993). In our concept, each cardiac cell is understood as an ensemble of independent functional units acting analogically to the network of atoms in laser active medium, constituted of a network of independent nodes, each node involving a set of discrete energy levels and transition probabilities between them. Quantum-like behaviour of the whole network is based on the interaction between the neighbouring nodes, which is given by the threshold-limited energy transfer leading to quantum-like behaviour of the whole network.
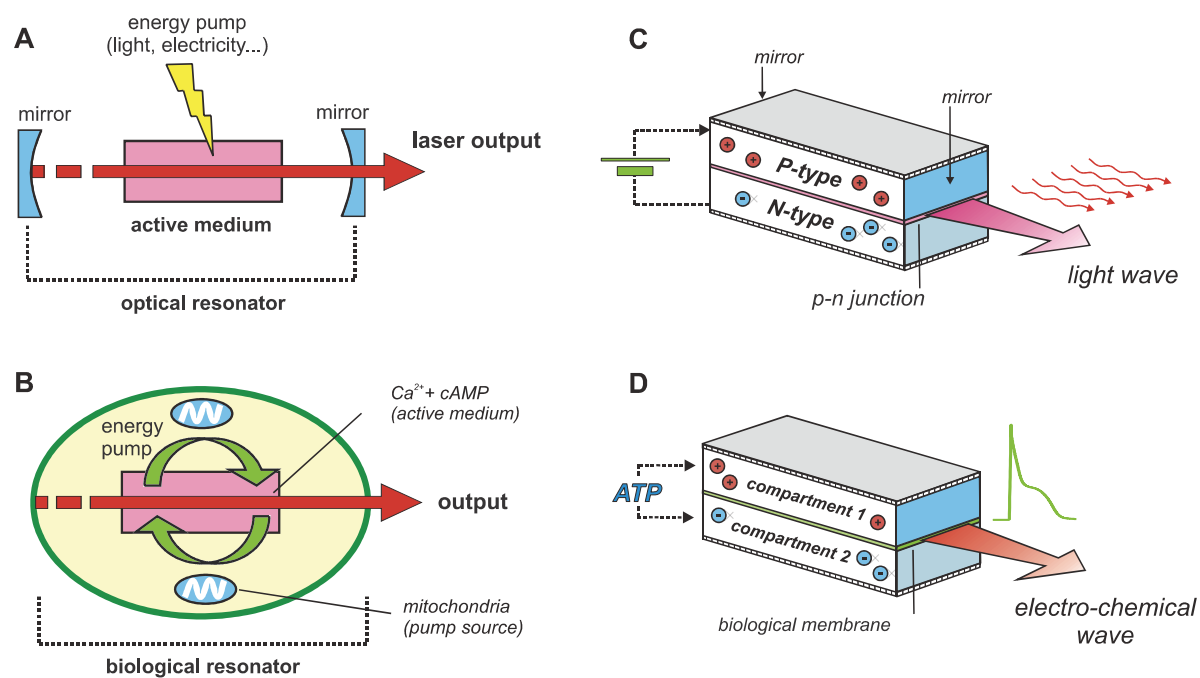

Fig. 4. Comparison of the function principle of the laser $(\mathrm{A}, \mathrm{C})$ and the cardiac pacemaker cell (B,D).

We have based this new concept of coherent resonant properties of the heart cells on an analogy with lasers - practical implementations of quantum resonators (Fig. 4) - in order to underline its advantages for the best energy efficiency of these cells. The LASER (Light Amplification by Stimulated Emission of Radiation) is a well-known device producing an intense monochromatic beam of coherent light, engineered on the principles of the quantum mechanics. The device uses a resonant cavity to induce light amplification and produce a coherent light output. First laser was constructed by Theodore Maiman in 1960, based on the original work of Albert Einstein and the groups of Townes/Basov and Prokhorov (reviewed in (Hecht, 2010)). Electromagnetic waves (such as its best known representation - light) have proven capacity to resonate according to the original work of Schrödinger (Schrödinger, 1933). When properly used, resonant cavity can generate coherent light waves, allowing creation of the laser.

In lasers, the presence of an active lasing medium is a key factor in their functioning (Fig. $\mathbf{4 A}, \mathbf{C})$. The particles of such lasing medium become progressively excited under continual 
optical pumping, resulting in their increasing number residing in higher-energy quantum states (described in details in (Chorvat, Jr. \& Chorvatova, 2008)). Consequently, when the number of particles in the excited state exceeds the number of particles in the ground state, the state of population inversion is achieved. In this case, the active medium acts as an optical amplifier, where the intensity of the light passing through it rises, instead of being absorbed. In addition to the requirements for a defined atomic or molecular structure of the laser's active medium, carefully-designed geometry (an optical resonator) needs to be employed to effectively combine the processes of spontaneous emission and light amplification by stimulated emission. As a result, in the active medium, a spontaneous emission of photons followed by the phenomenon of light amplification based on the process of stimulated emission takes place - which represents the basis of the laser. The resulting light output has unique properties which include directionality, monochromaticity, high power and coherence.

In cardiomyocytes, we believe that "active medium" of a biological laser-like resonator (Fig. 4B) results from stochastic behaviour of ionic channels (Chorvat, Jr. \& Chorvatova, 2008), namely the voltage-gated calcium ones (Junge, 1992). Opening of these channels in response to an action potential (AP) in a small microdomain between the transverse (T)-tubule and a sarcoplasmic reticulum (SR), also called a dyad, triggers process of calcium-induced calcium release (CICR), which underlies excitation-contraction coupling in cardiac cells (Bers, 2002;Niggli \& Egger, 2002) and is responsible for the contraction of the heart cell. In an analogy with the laser, the process of energy "absorption" is related to pushing the biological system into a higher energy state, while "emission" can be understood as return from such disequilibrium. In cardiac cells, we believe that the "absorption" relates to the active transport of ions - their removal from the cytosol. This process happens as active extrusion of ions such as calcium through the membrane out of the cytosol, using effective means of transportation such as $\mathrm{Na} / \mathrm{Ca}$ exchanger (Kimura et al., 1986) on one hand and, on the other hand, the compartmentalization of calcium ions into SR intracellular stores using adenosine trisphosphate (ATP)-powered SR calcium pumps (SERCA). This allows creation of a 10,000-fold calcium gradient between the intracellular stores and the cytosol (Lehnart et al., 2004), thus constituting a thermodynamically-unstable disequilibrium. Once this dynamic disequilibrium is in place, the process of "emission" can take place, which represents, in fact, an active process of return from such disequilibrium. In cardiac cells, this process can be understood either as a "spontaneous emission" process, witnessed by unitary calcium release (calcium sparks), or as "stimulated emission", triggered by stochastic opening of calcium channels and leading to CICR.

In this setting, the "population inversion"-like phenomena would take place in one node of a cardiac cell, which corresponds to several dyadic spaces between two t-tubules and the contractile apparatus, once the number of nodes in the "ready-to-fire" state becomes greater than the number of nodes with equilibrated calcium concentration. In this state only, the cardiac cell can achieve the capacity to sequentially amplify the calcium response occurring in one node to neighbouring nodes, and thus over to the whole cell and/or to the cardiac tissue. This process, macroscopically described as a continuous calcium wave, can be seen as sequential "stimulated emission" phenomena, with interaction between neighbouring nodes driven by threshold-limited energy transfer of a quantum nature.

In brief, our concept implies that in contractile cardiac myocytes, stochastic calcium release and the unitary properties of ionic channels stimulated during each excitation-contraction coupling cycle create "population inversion" and "spontaneous emission" phenomena, 
analogical to laser active medium (Chorvat, Jr. \& Chorvatova, 2008). Such medium, when powered by an incoming threshold-reaching voltage discharge in the form of an AP, responds to the calcium influx through L-type calcium channels by stimulated emission of $\mathrm{Ca}^{2+}$ ions in a coherent, synchronized and amplified CICR process. In this setting, molecular amplification stimulated by phosphorylation in protein cascades adds tuneable features to cardiac cells. The energy thus generated in cardiac cells is used for the mechanical work change in the conformation of a cell contractile apparatus - that results in the cell shortening and thus the whole heart contraction. Consequently, we propose that the heart functions as a coherent network of synchronously-firing cardiac myocytes behaving as amplifying blocks, coupled to pulse-generating pacemakers, acting as master-oscillators, all cooperating in a coherently-resonating cellular network under the hormonal control of the brain - the central regulator and control system, thus acquiring capacity to behave as a highly efficient pump expulsing the blood with smallest energy requirements. Advantages of the concept of a cardiac cell as a quantum resonator include high energy efficiency, robustness and selfcontrol.

\subsection{Pacemakers: Pulse generation based on coherent resonant properties of heart cells}

To design a pulse-generating system from an engineering point of view, precise interplay between several nonlinear effects must be reached, leading to the creation of short pulses, stable operation and high power efficiency. For example, in a laser, different techniques can achieve pulse generation, two of the most common being mode-locking and qualityswitching. We have previously described these processes in details (Chorvat, Jr. \& Chorvatova, 2008), where we also draw an analogy between different pulse-generating modes in a laser and those in the heart (Fig. 4B,D). Overall, no precise protein is responsible for cardiac rhythm; this mechanism is rather integrated at the cellular level (Noble, 2006). Cardiac tissue is known to be a highly non-linear medium that can support various complex rhythmic activities. To synchronize propagation between different cells in the heart, coherence of this firing is crucial, which means that it needs to be synchronous, but also delayed in time.

The rhythmic electrical activity - an inherent property of the heart - is initiated by the pacemakers in the sino-atrial (SA) node (Boyett et al., 2000). Pacemakers are the only cells in the heart that are capable of generating endogenous pulsed oscillations, thanks to spontaneous changes in membrane ionic permeability and specialized currents, particularly, pacemaker "funny" I $\mathrm{f}_{\mathrm{f}}$ current (Brown et al., 1979;DiFrancesco, 1993;DiFrancesco, 2006), thus governing heart-beating frequency (Noble, 2006). From an engineering point of view, $\mathrm{I}_{\mathrm{f}}$ current seems an ideal candidate for a quality-switching (Q-switch), which - in a laser - is based on a preventive feedback into the active medium while the laser medium is pumped. Consequently, as laser power increases, population inversion is generated, but without triggering the stimulated emission and increasing energy is stored in the active medium. At the opportune moment, the Q-switch device switches on, allowing an efficient extraction of the stored energy by highly-power pulse generation. Control of membrane depolarization via presence of high levels of basal cAMP and its attendant protein kinase A (PKA) phosphorylation (Bridge et al., 2006) point to their possible role in medium saturation and thus the control of firing properties of pacemakers in analogy with the creation of a "saturable absorber". In pacemakers, in contrast to ventricles, phosphorylation levels control the firing properties, with calcium having rather the tuning role allowing better internal 
control and flexibility. Thereby, the inherent, pulse-generating, pacemaking mechanism of the heart SA node can be regarded as a pulse-generating "master-oscillator".

Pulse-generating, pacemaker "master-oscillators" synchronize functioning of ventricular cells, which behave as a network of resonating structures constituted from a series of multipass amplifier components using energetically-efficient synchronisation tools, which allow the heart to function as a cell network, such as phase-locking. Phase-locking is an effect when several subunits are synchronized together in oscillatory behaviour with a coherent phase. Indeed, phase-locking, period-doubling bifurcations were previously proposed for a cardiac oscillator (Glass, 1991). To translate the signal towards neighbouring cells, cardiac myocytes employ electrically coupled junctional channels, permeable to small cytoplasmic molecules and ions, such as calcium, called gap junctions and/or narrow junctional clefts. Electrical field and gap junctional mechanisms act in concert to improve and stabilize the propagation of cardiac muscles (Sperelakis, 2003), resulting in synchronized responsiveness of the entire network of cells. Gap junctions are thereby important contributors to the unidirectionality of wave propagation as well as its synchronization between cells. Strong gap-junctional coupling has been proposed to synchronize the electrical oscillations of cells, while weak coupling has instead the capacity to phase-lock two cells (Sherman \& Rinzel, 1992). The heart has also been proposed to be a network of dynamically-coupled nonlinear oscillators from the metabolic point of view (Aon et al., 2006).

In the heart, pulse-generating pacemaker cells are electrically connected to neighbouring cells, thus allowing the synchronized propagation of oscillations within heart tissue. Once the pulse is generated in pacemaker cells, cardiac myocytes are then synchronized during each heart beat by voltage discharges in the form of $\mathrm{AP}$, based on the flow of electric current (Junge, 1992), which induces depolarization and subsequent contraction of cardiac myocytes. AP is a threshold process, result of a careful interplay between voltage-sensitive sodium and potassium ionic channels (Junge, 1992;Nerbonne \& Kass, 2005;Noble, 1962), which can be viewed in an analogy to an electronic design known as gain switching, which is at the basis of the creation of pulsed lasers. In this process, optical gain is negative when carrier density or pump intensity in the active region of the device is below the threshold, and switches to a positive value when the lasing threshold is exceeded. These characteristics are very comparable to the properties of APs, which switch the cell membrane potential from negative values (of around $-75 \mathrm{mV}$, when the cell is at its resting potential, below the threshold for AP activation) to positive values (to about $+40 \mathrm{mV}$, the Nernst equilibrium potential of sodium ions).

In addition, a number of molecular mechanisms perform regulatory tuning functions in the heart adding tunability features to cardiomyocyte "resonators". One of the most important is the hormonal control of ATP-powered protein phosphorylation, a well-recognized mode of signalling (Hata \& Koch, 2003) by protein cascades, initiated by G-protein stimulation. One example of such tuning in the heart is highly important $\beta$-adrenergic sympathetic regulation, which enhances cardiac function during stress and exercise performance (Movsesian, 1999). In pacemaker cells this regulation causes increases in cAMP production within cells, which, in turn, enhances "funny" $\mathrm{I}_{\mathrm{f}}$ current (DiFrancesco, 2006) and phosphorylates calcium channels, thus leading to an increase in the frequency of AP generation in these cells and, therefore, of heart beat. The modulation of calcium cycling by $\beta$-adrenergic receptor stimulation controls the strength of ventricular myocyte contraction and cardiac contractile reserves (Movsesian, 1999). Hormonal regulation acts as a general 
cell "tuning" mechanism with subsequent protein cascades as fine attenuators adjusting cell resonant properties.

Presented novel view of cardiac cells and pacemaker pulse-generation derived from the engineering-driven concept of biological quantum resonator opens new insights into understanding of heart functioning, thus allowing to comprehend several interrelated phenomena and their alteration in cardiovascular diseases. The concept brings a new viewpoint on cardiac diseases as possible alterations of cell resonant properties. In disease, disturbance of these features will first lead to adaptive remodelling, trying to restore the biological resonator, followed by the replacement of individual functions. However, if the repair mechanisms are not sufficient, the system will reach a state with distorted lasing properties, culminating in a non-linear energy collapse. It also points to the fact that to achieve efficient pharmaceutical treatment in such a complex environment, investigating the effects of medications on cell resonant properties is desirable as a signature of their energy efficiency. Last, but not least, deeper knowledge of cellular properties can thus be further translated into conceptual guidelines for the development of new emerging laser and optoelectronic technologies.

\subsection{Heart disease from the viewpoint of alteration of coherent resonant properties of cardiac cells}

Proposed concept of cardiac cells behaving as biological resonators brings a new viewpoint on cardiac diseases as possible alterations of their coherent resonant properties. Normal heart function can be seen as precisely-tuned, highly energetically-effective synchronous firing of the network of cardiomyocytes, each behaving as a biological resonator. In this setting, each cardiac cell functions with minimum energy to perform the required work. Consequently, the description of the heart functioning is based on the principle of harmony, as suggested previously for complex biological systems such as the heart (Noble, 2006), which emphasizes that some features, such as the pacemaker, are only observable in the state of precise balance of its components. At the same time, functioning involving precise resonant balance implicates that even a slight misalignment of components constituting the resonating system results in a significant drop in the system's energy efficiency, often in a complete loss of resonating properties.

In this regard, life-threatening conditions such as abnormal cardiac cell enlargement (hypertrophy) can be easily understood in the context of hypertrophy changing the size of the resonant cavity, preventing cells to sustain their resonant properties, leading to an increase in energy needs, loss of effectiveness and eventually heart failure (HF). For example, same set of proteins present in a cardiac cell, but with modified 3-dimensional topology could substantially alter its functioning. We have previously analyzed in details (Chorvat, Jr. \& Chorvatova, 2008), how can the HF - a cardiac disease touching large populations of patients - be described from the viewpoint of modifications of cardiac resonant properties.

We pointed out that typical features which accompany this disease can be understood as a failure of the biological resonator to achieve the "population inversion" state. Indeed, cardiac disease in general, and HF in particular, has been associated with increased occurrence of spontaneous calcium sparks, decreased cytosolic calcium transients and often diminished SR calcium load (Bers et al., 2003) due to reduced function of SERCA pumps, enhanced $\mathrm{Na} / \mathrm{Ca}$ exchange function and increased calcium leakage. These alterations have been proposed to lead to alteration of CICR. In addition, metabolic flexibility (Taegtmeyer $e t$ 
al., 2004), allowing the heart to switch from one substrate to another is severely reduced, leading to higher energy requirements in disease. Finally, synchronisation parameters are also affected: prolongation of AP duration is a characteristic feature of myocytes from diseased hearts (Hart, 1994) due to modifications of $\mathrm{K}^{+}$currents and $\mathrm{Ca}^{2+}$ handling. Cardiac hypertrophy or thickened heart muscle is a common hallmark of the progression of the disease. After adaptive myocardial remodeling (Gerdes, 2002), cardiac hypertrophy develops (Tamura et al., 1999), leading to congestive HF.

These findings suggest that cardiac cell in a failing heart exhibit features of 1) a rising "spontaneous emission" phenomena, vs. lowered "stimulated emission" ones; 2) the higher energy need, and 3) the overall loss of resonant and synchronisation properties. In this understanding, adaptive properties of cardiac muscles are put in place to restore the biological resonator capabilities of cells in their new environment, while maladaptive properties rather point to the incapacity of such restoration.

\subsection{Towards intelligent sensing of the dynamics in living systems: Resonance as a detection principle}

Resonance has a wide range of applications in technologies, particularly mass-media and other communication systems, but also nanotechnologies and information technologies. Radio is the oldest and to this day probably the best-known mass-media device which construction is based on resonance. Nikola Tesla was the first to demonstrate the feasibility of wireless communications in 1893, yet it was Guglielmo Marconi who developed the first workable radio communication and sent and received the first radio signal in Italy in 1895. Indeed, tuning to a preferred station equals choosing a resonance frequency. To this day, resonance remained a key technology governing the mass communication: in the last decades, nanoscale MicroElectroMechanical Systems (MEMS) oscillator/resonator technology has been greatly advanced and is now started to be employed in the cell phone and the consumer electronics industry (Saliterman, 2006). Its advantages are numerous, including small fabrication size and improved integration, possibility of simultaneous multifrequency use and finally the low power consumption; all these features predestine RF MEMS resonators to be used in portable applications.

Technologies based on resonance strongly advanced biomedical studies in the last decades. The extraordinary nature of resonance where the maximum response can be induced by minimum energy is indeed a holy-grail aim for any experimental method, promising ultrahigh sensitivity and specificity with minimum disturbance of the system under study. The resonance principle can be found in the roots of many macroscopic real-time 3D visualization techniques with one of the brightest examples being MRI (magnetic resonance imaging), which is exploiting the resonance of a nucleus of atoms absorbing energy from the magnetic field and is often used to image internal organs in medical diagnosis. On the other hand, resonance of light waves is mostly used for investigation of living cells and tissues and provide foundation of techniques such as optical tomography, speckle-interferometry, non-linear optical microscopy or FRET (Lauterborn et al., 2003;Periasamy \& Diaspro, 2003;Lakowicz, 2006; Verbiest et al., 2009;Sun et al., 2011). All these technologies take profit from a special capacity of resonance to improve the signal recognition from the studied phenomena while minimizing sample damage. Consequently they are now used daily in medicine and medical practice, although utilization of the resonance principle takes place in many cases unwittingly. 
In the future it is expected that resonance-based detection will be more and more widely used in study of living systems. In recent years, MEMS resonance biosensors are being implemented in the analytical laboratories to analyze the presence of molecules at a nanometric scale (Hillberg et al., 2005;Rosen \& Gurman, 2010). These are now used to isolate and identify stem cells, perform sensitive fingerprint sensor applications and are even intended to repair failing hearts (cardioMEMS, bioMEMS) (Gupta et al., 2010). Indeed, MEMS resonance biosensors have a wide range of applications from neural probes, blood analysis, to fabrication of endoscopes, as well as data storage. Another example is development of ultra sensitive biochemical sensors, based on surface plasmon resonance and resonant waveguide gratings, used to determine e.g. the affinities and kinetics of target analytes in a sample binding to the biological receptors immobilized on the sensor surface (Fang et al., 2006).

To improve the study of complex phenomena in living cells that we observe at the cellular and multicellular levels - such as the pacemaker - we now need to expand detection technologies and create resonance detectors capable of sensing resonance alterations in living systems at higher hierarchical levels. Currently, we apply deterministic approach in this investigation, which means that we characterize all entities from which such system is built. In other words, we cut the system into pieces and examine each piece in details (determining its anatomical design, or protein mapping). However, modelling of complex living systems, such as the heart, revealed fundamental limitations of such scientific investigations. Denis Noble (Noble, 2006) detailed serious limitations that are attached to the use of such "bottom-up" or "top-down" approach in the study of living systems and their functions, mainly linked to the failure of these approaches to examine what does create the bond between sub-systems to generate a new, higher hierarchical level. As a result, this approach is proving not to be enough: as an example, it is becoming clear that despite knowing the whole genome, we still cannot understand why a healthy system suddenly, in the middle of an (apparently healthy) lifetime, changes into a diseased one. We now start to understand that, instead of dissecting individual components of a complex living system, we rather need to study the system as a whole from its "centre" to comprehend the casual chain in the system.

Recent technologies, described above, allow detection of resonance states at the level of atoms and molecules. However, at the moment there are no such appropriate detection systems working at the level of whole cells or organs. More specifically, in the future we will need to design a new type of intelligent detectors, capable of deciphering the natural resonance frequencies and their changes in physiological and pathophysiological conditions to monitor complex phenomena, such as the pacemaker physiology. We need devices capable of capturing coherent behaviour resulting from interplay of each of its subcomponents at every hierarchical level. Search for new, multi-dimensional intelligent detection systems that would have capacity to observe the system and its behaviour in an observer-independent way, is the future of recording tools that would account for the complexity of the studied system and would therefore become sensors of its functionality, as well as of its alterations in diseased states.

\section{Conclusion}

Understanding complex phenomena in living systems is the challenge of the $21^{\text {st }}$ century. Despite searching for centuries for the best description of what life really is, we still lack 
precise understanding of this extraordinary phenomenon. Living systems function at a basis of a highly energetically-advantageous dynamic disequilibrium of oscillating components, organized in sub-systems. To create and maintain such system, it is therefore highly important that its components behave coherently. To achieve such behaviour, it is necessary to strengthen synchronized coupling between these components. We propose that the phenomenon of resonance can contribute to this synchronisation in a highly effective way. Fundamental for all oscillating systems, resonance is closely linked to energy usage and, with each force having resonant representation, this phenomenon has all the attributes to play a fundamental role in driving coherent behaviour in living systems. It can therefore also help us to comprehend such complex phenomena in living systems as is the pacemaker. Monitoring harmonious behaviour of the heart based on synchronous oscillations of cardiac cells, each functioning as a pulsed biological quantum resonator/amplifier, opens a new insight into understanding of pacemaking and thus of heart functioning at a synch. We are now beginning to understand, with heart beat being the first such example, that some cell features, such as its resonant properties, are only observable in the state of precise balance in a physiological state. This concept also incorporates the role of genes in re-creating, in the new environment, a state with the most appropriate biological resonant properties by tuning the cell resonator via the expression of precise protein clusters. Investigation of resonance properties can allow to comprehend not only the normal functioning of living cardiac cells, but also their alterations in a disease. Understanding cell resonant properties as a signature of their energy efficiency can also help to achieve an efficient pharmaceutical treatment by investigating effects of medications in such complex environment. We are convinced that resonant features are not likely to be unique to cardiac cells, but that resonance is a more generalized feature in living systems.

Nowadays, it is crucial to add an engineering point of view to the analysis of physiological phenomena. We have developed tools that allow us to decipher every component of the living system individually. Now we need to design new advanced tools that would allow to study functioning of the coherent behaviour of the living system in its dynamic complexity: detectors that would be able to monitor resonances in living systems at their different hierarchical levels. In parallel, deeper knowledge of cellular properties as biological quantum resonator can be further translated into design of new emerging resonance-based optoelectronic detection technologies.

\section{Acknowledgment}

We would like to acknowledge support from the EC's Seventh Framework Programme LASERLAB-EUROPE, grant agreement $\mathrm{n}^{\circ}$ 228334, and Research grant agency of the Ministry of Education, Science, Research and Sport of the Slovak Republic VEGA No. $1 / 0530 / 09$.

We would specially like to thank Jan Kodon (www.reasonance.org) for fruitful discussion.

\section{References}

Aon, M. A., Cortassa, S., \& O'Rourke, B. (2006). The fundamental organization of cardiac mitochondria as a network of coupled oscillators. Biophys.J 91, 4317-4327.

Bateson, W. (1894). Materials for the study of variation, reprinted 1992 ed. Johns Hopkins Press, Baltimore. 
Bateson, W. (1913). Problems of genetics, reprinted 1979 ed., Yale University Press, New Haven.

Bers, D. M. (2002). Cardiac excitation-contraction coupling. Nature 415, 198-205.

Bers, D. M., Eisner, D. A., \& Valdivia, H. H. (2003). Sarcoplasmic reticulum Ca2+ and heart failure: roles of diastolic leak and Ca2+ transport. Circ.Res. 93, 487-490.

Bloch, S. C. (1997). Introduction to classical and quantum harmonic oscillators, reprinted 2001 ed., pp. 1-363. A Wiley-Interscience, NY, U.S.A.

Bohm, D. (1951). Quantum Theory, reprinted 1989 ed., pp. 1-646. Dover Publications, NY, U.S.A.

Boyett, M. R., Honjo, H., \& Kodama, I. (2000). The sinoatrial node, a heterogeneous pacemaker structure. Cardiovasc.Res. 47, 658-687.

Bridge, J. H., Davidson, C. J., \& Savio-Galimberti, E. (2006). A novel mechanism of pacemaker control that depends on high levels of cAMP and PKA-dependent phosphorylation: a precisely controlled biological clock. Circ.Res. 98, 437-439.

Brown, H. F., DiFrancesco, D., \& Noble, S. J. (1979). How does adrenaline accelerate the heart? Nature 280, 235-236.

Chladni, E. (1787). Entdeckungenu"ber die Theorie des Klanges [Discoveries concerning the theory of sound], pp. 1-100. Leipzig.

Chorvat, D., Jr. \& Chorvatova, A. (2008). Cardiac cell: a biological laser? Biosystems 92, 49-60.

Davies, P. (2005). A quantum recipe for life. Nature 437, 819.

Davies, P. C. (2004). Does quantum mechanics play a non-trivial role in life? Biosystems 78, 69-79.

DiFrancesco, D. (1993). Pacemaker mechanisms in cardiac tissue. Annu.Rev.Physiol 55, 455472.

DiFrancesco, D. (2006). Serious workings of the funny current. Prog.Biophys.Mol.Biol. 90, 1325.

Eckhardt, B., Ott, E., Strogatz, S. H., Abrams, D. M., \& McRobie, A. (2007). Modeling walker synchronization on the Millennium Bridge. Phys.Rev.E Stat.Nonlin.Soft.Matter Phys. $75,021110$.

Fang, Y., Ferrie, A. M., Fontaine, N. H., Mauro, J., \& Balakrishnan, J. (2006). Resonant waveguide grating biosensor for living cell sensing. Biophys.J 91, 1925-1940.

Foster R \& Kreitzman L (2004). Rhythms of Life, pp. 1-276. Profiles Books, Ltt, London.

Gerdes, A. M. (2002). Cardiac myocyte remodeling in hypertrophy and progression to failure. J.Card Fail. 8, S264-S268.

Glass, L. (1991). Nonlinear dynamics of physiological function and control. Chaos. 1, 247-250.

Gupta, K., Kim, D. H., Ellison, D., Smith, C., Kundu, A., Tuan, J., Suh, K. Y., \& Levchenko, A. (2010). Lab-on-a-chip devices as an emerging platform for stem cell biology. Lab Chip. 10, 2019-2031.

Hameroff, S., Nip, A., Porter, M., \& Tuszynski, J. (2002). Conduction pathways in microtubules, biological quantum computation, and consciousness. Biosystems 64, 149-168.

Hart, G. (1994). Cellular electrophysiology in cardiac hypertrophy and failure. Cardiovasc.Res. 28, 933-946.

Hata, J. A. \& Koch, W. J. (2003). Phosphorylation of G protein-coupled receptors: GPCR kinases in heart disease. Mol.Interv. 3, 264-272.

Hecht, J. (2010). A short history of laser development. Appl.Opt. 49, F99-122. 
Hillberg, A. L., Brain, K. R., \& Allender, C. J. (2005). Molecular imprinted polymer sensors: implications for therapeutics. Adv.Drug Deliv.Rev. 57, 1875-1889.

Junge, D. (1992). Nerve and muscle excitation, 3 ed., pp. 1-263. Sinauer Associates, Inc. U.S.A., Sunderland, Massachusetts.

Kimura, J., Noma, A., \& Irisawa, H. (1986). Na-Ca exchange current in mammalian heart cells. Nature 319, 596-597.

Lakhovsky, G. (1929). The Secret of Life, reprinted in 2007 ed., pp. 1-201. A Digireads.com Publishing, Stilwell, U.S.A.

Lakowicz, J. R. (2006). Principles of Fluorescence Spectroscopy, 3 ed., pp. 1-923. Springer, New York.

Lauterborn, W., Kurz, T., \& Wiesenfeldt, M. (2003). Coherent Optics: Fundamentals and Applications, pp. 1-333. Springer-Verlag, Berlin Heidelberg.

Lehnart, S. E., Wehrens, X. H., Kushnir, A., \& Marks, A. R. (2004). Cardiac ryanodine receptor function and regulation in heart disease. Ann.N.Y.Acad.Sci. 1015, 144-159.

McDonnell, M. D. \& Abbott, D. (2009). What is stochastic resonance? Definitions, misconceptions, debates, and its relevance to biology. PLoS.Comput.Biol. 5, e1000348.

McFadden, J. (2000). Quantum Evolution, pp. 1-338. Norton\&Company Ltd., NY, U.S.A.

McFadden, J. \& Al Khalili, J. (1999). A quantum mechanical model of adaptive mutation. Biosystems 50, 203-211.

Montagnier, L., Aissa, J., Ferris, S., Montagnier, J. L., \& Lavallee, C. (2009). Electromagnetic signals are produced by aqueous nanostructures derived from bacterial DNA sequences. Interdiscip.Sci. 1, 81-90.

Moss, F., Ward, L. M., \& Sannita, W. G. (2004). Stochastic resonance and sensory information processing: a tutorial and review of application. Clin.Neurophysiol. 115, 267-281.

Movsesian, M. A. (1999). Beta-adrenergic receptor agonists and cyclic nucleotide phosphodiesterase inhibitors: shifting the focus from inotropy to cyclic adenosine monophosphate. J.Am.Coll.Cardiol. 34, 318-324.

Murray, J. (1993). Mathematical biology, 2 ed., Springer-Verlag, Berlin.

Nerbonne, J. M. \& Kass, R. S. (2005). Molecular physiology of cardiac repolarization. Physiol Rev. 85, 1205-1253.

Niggli, E. \& Egger, M. (2002). Calcium quarks. Front Biosci. 7, d1288-d1297.

Noble, D. (1962). A modification of the Hodgkin--Huxley equations applicable to Purkinje fibre action and pace-maker potentials. J Physiol 160, 317-352.

Noble, D. (2004). Modeling the heart. Physiology (Bethesda.) 19, 191-197.

Noble, D. (2006). The music of life. Biology beyond the genome., 1 ed., pp. 1-153. Oxford University Press, Inc., New York, U.S.A.

Penrose, R. (2001). Consciousness, the brain, and spacetime geometry: an addendum. Some new developments on the Orch OR model for consciousness. Ann.N.Y.Acad.Sci. 929, $105-110$

Periasamy, A. (2001). Fluorescence resonance energy transfer microscopy: a mini review. J Biomed.Opt. 6, 287-291.

Periasamy, A. \& Diaspro, A. (2003). Multiphoton microscopy. J Biomed.Opt. 8, 327-328.

Periasamy, A., Wallrabe, H., Chen, Y., \& Barroso, M. (2008). Chapter 22: Quantitation of protein-protein interactions: confocal FRET microscopy. Methods Cell Biol. 89, 569598. 
Rabi, I. I., Zacharias, J. R., Millman, S., \& Kusch, P. (1992). Milestones in magnetic resonance: 'a new method of measuring nuclear magnetic moment'. 1938. J Magn Reson.Imaging 2, 131-133.

Rosen, Y. \& Gurman, P. (2010). MEMS and microfluidics for diagnostics devices. Curr.Pharm.Biotechnol. 11, 366-375.

Saliterman, S. S. (2006). Fundamentals of BioMEMS and Medical Microdevices, pp. 1-608. SPIE Press Book, London, UK.

Schrödinger, E. (1933). Mémoires sur la mécanique ondulatoire, reprinted 1988 ed., pp. 1-234. Jacques Gabay, Paris.

Schrödinger, E. (1944). What is life?, reprinted 2006 ed., pp. 1-184. Cambridge University Press, Cambridge.

Sherman, A. \& Rinzel, J. (1992). Rhythmogenic effects of weak electrotonic coupling in neuronal models. Proc.Natl.Acad.Sci.U.S.A 89, 2471-2474.

Sperelakis, N. (2003). Combined electric field and gap junctions on propagation of action potentials in cardiac muscle and smooth muscle in PSpice simulation. J Electrocardiol. 36, 279-293.

Strogatz, S. H. (2003). Sync: the emerging science of spontaneous order, pp. 1-338, Penguin Books, London, UK.

Strogatz, S. H. \& Stewart, I. (1993). Coupled oscillators and biological synchronization. Sci.Am. 269, 102-109.

Sun, Y., Wallrabe, H., Seo, S. A., \& Periasamy, A. (2011). FRET Microscopy in 2010: The Legacy of Theodor Forster on the 100th Anniversary of his Birth. Chemphyschem. 12, 462-474.

Taegtmeyer, H., Golfman, L., Sharma, S., Razeghi, P., \& van Arsdall, M. (2004). Linking gene expression to function: metabolic flexibility in the normal and diseased heart. Ann.N.Y.Acad.Sci. 1015, 202-213.

Tamura, T., Said, S., \& Gerdes, A. M. (1999). Gender-related differences in myocyte remodeling in progression to heart failure. Hypertension 33, 676-680.

Valone, T. (2002). Harnessing the Wheelwork of Nature: Tesla's Science of Energy, pp. 1-288. Adventures Unlimited Press, Kempton, U.S.A.

Verbiest, T., Clays, K., \& Rodriguez, V. (2009). Second-order Nonlinear Optical Characterization Techniques: An Introduction, pp. 1-192. CRC Press Online.

Weiss, K. (2002). Good vibrations: the silent symphony of life. Evolutionary Anthropology 11, 176-182.

Wiesenfeld, K. \& Jaramillo, F. (1998). Minireview of stochastic resonance. Chaos. 8, 539-548.

Winfree, A. T. (1980). The Geometry of Biological Time, reprinted 2001 ed., pp. 1-777. SpringerVerlag, NY, Berlin, Heidelberg. 


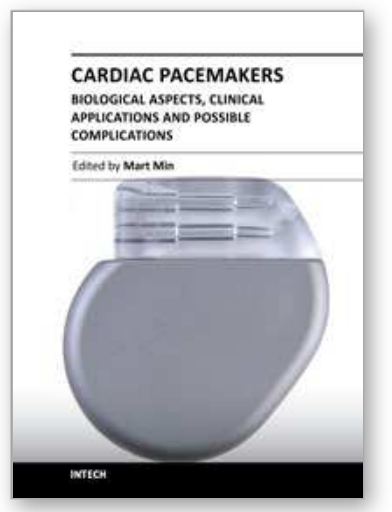

\section{Cardiac Pacemakers - Biological Aspects, Clinical Applications and Possible Complications}

Edited by Prof. Mart Min

ISBN 978-953-307-639-3

Hard cover, 194 pages

Publisher InTech

Published online 06, September, 2011

Published in print edition September, 2011

Clinical usage of artificial pacing dates back to 1958, when the battery powered cardiac pacemakers became available. Modern implantable pacemakers are the complicated electronic devices operating 10 years continuously without battery exchange. Though the development of devices is not a primary topic of the book, certain efforts towards developing of biologic pacemakers through tissue engineering and studying of cell synchronization are discussed. The main attention is paid to implementations of pacemakers in different medical situations oriented towards widening the clinical indications for implanting the cardiac pacemakers. New methods and devices in cardiac resynchronization therapy (CRT) have received particular attention. Placing of pacing electrodes has been treated soundly. Furthermore, emerging of complexities and complications in new clinical situations and other safety problems have been discussed thoroughly. The authors have derived the used information from their own clinical practice and experiences of their medical colleagues. These and other pragmatic features can be acknowledged as the most valuable asset of the book.

\section{How to reference}

In order to correctly reference this scholarly work, feel free to copy and paste the following:

A. Chorvatova and D. Chorvat Jr (2011). Coherent Resonant Properties of Cardiac Cells, Cardiac Pacemakers - Biological Aspects, Clinical Applications and Possible Complications, Prof. Mart Min (Ed.), ISBN: 978-953307-639-3, InTech, Available from: http://www.intechopen.com/books/cardiac-pacemakers-biological-aspectsclinical-applications-and-possible-complications/coherent-resonant-properties-of-cardiac-cells1

\section{INTECH}

open science | open minds

\section{InTech Europe}

University Campus STeP Ri

Slavka Krautzeka 83/A

51000 Rijeka, Croatia

Phone: +385 (51) 770447

Fax: +385 (51) 686166

www.intechopen.com

\section{InTech China}

Unit 405, Office Block, Hotel Equatorial Shanghai

No.65, Yan An Road (West), Shanghai, 200040, China

中国上海市延安西路65号上海国际贵都大饭店办公楼 405 单元

Phone: +86-21-62489820

Fax: $+86-21-62489821$ 
(C) 2011 The Author(s). Licensee IntechOpen. This chapter is distributed under the terms of the Creative Commons Attribution-NonCommercialShareAlike-3.0 License, which permits use, distribution and reproduction for non-commercial purposes, provided the original is properly cited and derivative works building on this content are distributed under the same license. 